\title{
Timing of lymphadenectomy during robot-assisted radical cystectomy: before or after cystectomy? Fifteen cases with totally intracorporeal urinary diversions
}

\author{
Mehmet Salih Boga, Mutlu Ates \\ Department of Urology, Antalya Training and Research Hospital, Health Sciences University, Antalya, Turkey
}

Videosurgery Miniinv 2020; 15 (4): 596-601

DOI: https://doi.org/10.5114/wiitm.2020.93793

\begin{abstract}
Introduction: Many publications detail the level and number of lymphadenectomies, whereas the timing of pelvic lymph node dissection (PLND) is infrequently discussed in the robot-assisted radical cystectomy (RARC) series.

Aim: To determine the effects of performing PLND before or after cystectomy in totally intracorporeal RARC on operative outcomes.

Material and methods: A total of 15 patients included in the study underwent RARC and intracorporeal orthotopic neobladder. Of these, 8 patients underwent PLND before cystectomy (group 1), whereas 7 underwent PLND after cystectomy (group 2). Demographic information, intraoperative data, and post-operative outcomes were recorded for each patient.

Results: The mean $\pm S D$ age was $61.87 \pm 6.76$ years. Overall mean operation time (OT) was $537.33 \pm 63.07$ min. The mean EBL and hospitalization time were $322.33 \pm 69.92 \mathrm{ml}$ and $13.87 \pm 5.2$ days. The number of $L N$ removed was $23.75 \pm 3.454$ for group 1 and $13.71 \pm 6.873$ for group $2(p=0.007)$. The postoperative pathological stages were: pT2(5), pT3(2), pT4(1) for group 1, pT2(4), pT3(2), pT4(1) for group 2. Surgical margins were negative in all patient. Overall complications occurred in 7 (46.6\%) patients. Only the number of $L N$ removed was statistically significant in favor of group 1 ( $p=0.007)$. Mean follow-up was 15.87 months.

Conclusions: Our initial experience with performing extended PLND (ePLND) before or after cystectomy in totally intracorporeal RARC appears to be favorable, with similar oncological results and acceptable complication rates. However, although the number of cases is too low for statistical evaluation, it seems to be advantageous to perform ePLND before cystectomy in terms of LN number and operation time.
\end{abstract}

Key words: bladder tumor, cystectomy, robotic, intracorporeal neobladder, extended pelvic lymph node dissection, timing of lymphadenectomy.

\section{Introduction}

Radical cystectomy (RC) with lymphadenectomy is the gold standard treatment method in terms of local control and long-term disease-free survival in muscle-invasive or high-risk non-muscle invasive bladder tumors [1]. Although the survival outcomes of open radical cystectomy (ORC) and robot-assisted radical cystectomy (RARC) are similar [2], the use of RARC is increasing day by day due to its advantages such as less bleeding, earlier bowel motility and shorter length of stay [3]. However, the reported total intracorporeal RARC series are still limited [4].

Publications strongly suggest that all patients undergoing radical cystectomy for bladder cancer 
should undergo concomitant extended pelvic lymph node dissection (ePLND) because of the positive effect on correct staging and survival [5]. Although controversy surrounds PLND levels, many researchers agree that ePLND shows superior survival rates and correct staging compared to limited PLND [6, 7] and superextended PLND provides no additional survival benefits due to the potential for increased operation time and patient morbidity [8]. In addition, it is reported that meticulous dissection is more important than the number of nodes removed $[7,8]$

In the RC series, there are many publications on the level and number of lymph nodes removed including discussion about whether lymph nodes should be removed separately or en bloc, whereas the timing of PLND in RC is very rare. While there is a publication on the timing of PLND in the open $\mathrm{RC}$ series, according to our knowledge, there are no reported studies on the timing of PLND in RARC.

\section{Aim}

The aim of this study is to present our initial experience with performing ePLND before or after RC in totally intracorporeal RARC and its effect on complications, operation time and number of removed lymph nodes.

\section{Material and methods}

Robotic surgery has been performed at our center since January 2015. Intracorporeal RC began after over 100 robotic radical prostatectomies and approximately 20 cases of robotic radical cystectomy and open ileal loop/orthotopic application.

The current study included 15 patients who underwent RARC and totally intracorporeal neobladder for invasive bladder cancer between August 2017 and February 2019. All patients underwent ePLND, for which margins were determined previously by a protocol [9]. All procedures were completed successfully without open conversion. Pathologic staging was assigned according to the 2016 WHO tumor-node-metastasis classification [10].

Of the 15 patients included in the study, 8 underwent ePLND before cystectomy (group 1), whereas 7 underwent ePLND after cystectomy (group 2). In the first group, unlike the standard technique the operation was initiated with extended lymphadenectomy before cystectomy followed by ureter and seminal vesicle dissection. With this technique, the lymph nodes were removed in one piece as an enbloc procedure with the bladder, in accordance with anatomical structure. In the second group, PLND was performed classically after cystectomy.

Demographic information, intraoperative data, and post-operative complications were recorded for each patient. The groups were compared according to the duration of operation, number of lymph nodes removed, oncologic outcomes and complication rates.

\section{Surgical technique}

All patients underwent RARC, ePLND, and totally intracorporeal urinary diversion using the da Vinci Xi robotic system (Intuitive Surgical, CA, USA). Studer's neobladder technique was used as a diversion procedure [11]. Cystectomy was performed using the method described previously [12].

In the current study, ePLND was performed, unconventionally, before cystectomy. Following dissection of the ureter and seminal vesicles, we removed nodes between the aortic bifurcation and common iliac vessels proximally, the genitofemoral nerve laterally, the circumflex iliac vein distally, and the internal iliac vessels posteriorly; these tissues were not removed in pieces but removed as a whole. Then, as the pedicle and tissue plans were clearly visible, the bladder branches of the internal iliac artery were controlled and, after dissection of the bladder, the dissected bilateral lymph nodes were sent to pathology as a whole with the bladder. The surgical technique is shown in a supplemental video.

\section{Statistical analysis}

Statistical analysis was performed with SPSS, v.23.0 statistical software (IBM SPSS Statistics for Windows, Version 23.0. Armonk, NY: IBM Corp.). The $\chi^{2}$ test was used to determine whether the distributions of categorical variables were different across groups. Categorical variables are described by frequencies and percentages. Continuous variables are presented as mean and standard deviations. Independent samples $t$-test was used for the comparison of continuous variables among groups. A $p$-value $<0.05$ is considered statistically significant.

\section{Results}

Mean \pm SD patient age was $61.87 \pm 6.760$ years. Of the 15 patients, 14 were men and 1 was a wom- 
an. Overall mean operation time (OT) was 537.33 $\pm 63.07 \mathrm{~min}$. OT was shorter in group 1 than group 2, although not statistically significant (515.00 \pm 75.024 min vs. $562.86 \pm 35.923 \mathrm{~min}$, group 1 and group 2, respectively, $p=0.138$ ). The estimated blood loss (EBL) was $313.13 \pm 69.124 \mathrm{ml}$ for group 1 and 332.86 $\pm 74.769 \mathrm{ml}$ for group $2(p=0.607)$. The lodge drain was removed at a mean of $10.00 \pm 1.89$ days ( 9.63 \pm 2.13 days for group 1 and $10.43 \pm 1.61$ days for group 2, $p=0.423)$. All procedures were completed successfully without open conversion. Demographic information and operative parameters of the patients were similar for both groups (all $p$-values $>0.05$, except follow-up time). Mean follow-up was 15.87 months $(14.75 \pm 2.37$ months for group 1 and $17.14 \pm 0.69$ months for group $2, p=0.025)$. Patient demographics and operative parameters are shown in Table I.

The mean number of lymph nodes removed was $23.75 \pm 3.454$ in group 1 and $13.71 \pm 6.873$ in group $2(p=0.007)$. Pathological stages were found - pT2 (5 patients), pT3 (2), pT4 (1) (prostatic invasion) - in cystectomy specimens, and 3 patients had positive lymph nodes for group 1. pT2 (4), pT3 (2), pT4 (1), and 2 lymph nodes were positive for group 2 .

Postoperative complications occurred in 7 (46.6\%) patients in the perioperative early (0-30 days) period; 5 out of 7 (71.43\%) were minor (Clavien 1-2), 2 (28.53\%) were major (Clavien 3-5) complications. Group 1 contained 1 patient with Clavien 1 (wound infection), 1 patient with Clavien 2 (underwent intravenous antibiotic treatment for complicated urinary tract infection), and 1 patient with
Clavien 4 (complicated urinary infection and three times dialysis due to acute renal failure) complications. Group 2 contained 1 patient with Clavien 1 (wound infection), 2 patients with Clavien 2 (one underwent intravenous antibiotic treatment due to complicated urinary infection, the other underwent total parenteral nutrition due to ileus), and 1 patient with Clavien 3 (facial repair was performed with sedation and local anesthesia for serious discharge from the wound site and facial defects) complications. The frequency of complications was similar between the two groups ( $p=0.728)$. Postoperative pathologic parameters and complication rates are shown in Table II.

\section{Discussion}

Although the use of RARC has become more widespread, due to the difficulty of this procedure and its long operation time, the urinary diversion of RARC is generally performed extracorporeally. The number of centers that have performed totally intracorporeal urinary diversion is still limited.

Performing the whole procedure totally intracorporeally might lead to decreased insensible fluid loss from the bowels, which might also prevent the development of electrolyte imbalance, resulting in a quicker return of bowel function [13].

PLND is technically very difficult and takes a long time in RARC with intracorporeal urinary diversion. Although it has been shown in many studies that ePLND increases progression-free survival and overall survival in patients with or without lymph node

Table I. Patient demographics and operative parameters

\begin{tabular}{|lcccc|}
\hline Variables & $\begin{array}{c}\text { Group 1 } \\
\text { (mean } \pm \text { SD/\%) }\end{array}$ & $\begin{array}{c}\text { Group 2 } \\
\text { (mean } \pm \text { SD/\%) }\end{array}$ & $\begin{array}{c}\text { Overall } \\
(\text { mean } \pm \text { SD/\%) }\end{array}$ & $P$-value \\
\hline Number of patients & $8(53.3)$ & $7(46.7)$ & 15 & $14 / 1$ \\
\hline Sex M/F & $7 / 1$ & $7 / 0$ & $61.87 \pm 6.760$ & 0.608 \\
\hline Age [years] & $61.00 \pm 7.672$ & $62.86 \pm 5.984$ & $537.33 \pm 63.072$ & 0.138 \\
\hline $\begin{array}{l}\text { Mean operation time, } \\
\text { min } \pm \text { SD }\end{array}$ & $515.00 \pm 75.024$ & $562.86 \pm 35.923$ & $322.33 \pm 69.920$ & 0.607 \\
\hline EBL [ml] & $313.13 \pm 69.124$ & $332.86 \pm 74.769$ & $13.87 \pm 5.222$ & 0.714 \\
\hline Length of stay [day] & $13.38 \pm 5.125$ & $14.43 \pm 5.682$ & $10.00 \pm 1.890$ & 0.423 \\
\hline $\begin{array}{l}\text { Removal of lodge drain } \\
\text { time [days] }\end{array}$ & $9.63 \pm 2.134$ & $10.43 \pm 1.618$ & $15.87 \pm 2.134$ & 0.025 \\
\hline Follow-up & $14.75 \pm 2.375$ & $17.14 \pm 0.690$ & & \\
\hline
\end{tabular}


Table II. Postoperative pathologic parameters and complication rates

\begin{tabular}{|c|c|c|c|c|}
\hline Parameter & $\begin{array}{c}\text { Group } 1 \\
\text { (mean } \pm \text { SD/\%) }\end{array}$ & $\begin{array}{c}\text { Group } 2 \\
\text { (mean } \pm \text { SD/\%) }\end{array}$ & $\begin{array}{c}\text { Overall } \\
\text { (mean } \pm \text { SD/\%) }\end{array}$ & $P$-value \\
\hline End pathological stage, $n(\%)$ : & & & & 0.978 \\
\hline pTx & - & - & - & \\
\hline pTO & - & - & - & \\
\hline pT1 & - & - & - & \\
\hline pT2 & $5(62.5)$ & $4(57.1)$ & $9(60.0)$ & \\
\hline pT3 & $2(25.0)$ & $2(28.6)$ & $4(26.7)$ & \\
\hline pT4 & $1(12.5)$ & $1(14.3)$ & $2(13.3)$ & \\
\hline Number of lymph nodes resected, $n$ & $23.75 \pm 3.454$ & $13.71 \pm 6.873$ & $19.07 \pm 7.285$ & $0.007^{\star}$ \\
\hline N positivity, $n(\%)$ & $3(37.5)$ & $2(28.6)$ & $5(33.3)$ & 0.714 \\
\hline Complication Clavien, $n(\%)$ : & & & & 0.728 \\
\hline Clavien 1-2 (minor) & $2(25)$ & $3(42.9)$ & $5(33.3)$ & \\
\hline Clavien 3-5 (major) & $1(12.5)$ & $1(14.3)$ & $2(13.3)$ & \\
\hline
\end{tabular}

${ }^{*} P<0.05$ is considered statistically significant.

positivity [14], there is no recommendation as to whether extended PLND should be performed before or after RARC. Only one publication compared the timing of ePLND in open RC groups [15].

A study on the timing of ePLND in open RC revealed that the mean cystectomy time and mean total operation times were significantly shorter in the group with ePLND performed before cystectomy than the group with ePLND performed after cystectomy $(157.50 \pm 37.57 \mathrm{~min}$ vs. $190.07 \pm 49.55 \mathrm{~min}$, retrospectively, $p<0.001)$. They reported that performing ePLND before cystectomy shortens the total operation time via shortening particularly the cystectomy time. They explained that the ePLND before cystectomy fully skeletonizes the pelvic vascular structures, and isolation and control of pedicles during cystectomy is easier, which might be an explanation of shortening the cystectomy time.

Research on the new surgical technique is ongoing to decrease the operation time of RARC, although totally intracorporeal RARC series showed that the operation time was shortened with experience $[16,17]$. In our study, mean operation time was $537.33 \pm 63.072 \mathrm{~min}$. The total operation time in the group of ePLND performed before cystectomy was shorter than in the group of ePLND performed after cystectomy, although it was not statistically significant $(515.00 \pm 75.024 \mathrm{~min}$ vs. $562.86 \pm 35.923 \mathrm{~min}$, respectively, $p=0.138)$. These results were similar to some previous publications reported as the first experiences of robot-assisted intracorporeal neobladder series [18], but it was higer than some of center's result. Collins and Wiklund reported OT between 330-720 min in their review of the robot-assisted intracorporeal neobladder series [13]. Studies have shown that the operative time is reduced after the first 15 cases [19]. In the current paper we have presented outcomes of our first 15 RARC cases with totally intracorporeal urinary diversion. We believe that our operation time will decrease in future as in previous reported series from other centers.

Complication rates after RARC vary according to methods and patient selection in the literature $[20,21]$. Postoperative complications occurred in $7(46.6 \%)$ patients in our study, in the perioperative early (0-30 days) period; 5 out of 7 (71.43\%) were minor (Clavien 1-2), 2 (28.53\%) were major (Clavien 3-5) complications. Group 1 contained 2 patients with Clavien 1-2, 1 patient with Clavien 4 complications. Group 2 contained 3 patients with Clavien 1-2, 1 patient with Clavien 3 complications. The complication rates were comparable to contemporary robotic series [19, 22]. Most of the complications were low grade in our study. One major complication was reported in both groups.

Surgery techniques, complication rates and severity of complications are the important factors affecting LOS in hospital $[23,24]$. It has been shown in 
many studies that the LOS in RARC is shorter than ORC $[22,24]$. In our study, overall LOS was 13.87 \pm 5.222 days. It was $13.38 \pm 5.125$ days in the group of ePLND performed before cystectomy and 14.43 \pm 5.682 days in the group of ePLND performed after cystectomy $(p=0.714)$. The lodge drain was removed at a mean of $10.00 \pm 1.89$ days $(9.63 \pm 2.13$ days for group 1 and $10.43 \pm 1.61$ days for group 2 , $p=0.423)$. The results of our study were comparable to previous RARC series performed totally intracorporeally [19].

Thress et al. evaluated the outcomes of ileal conduit and neobladder patients in totally intracorporeal RARCs [17]. They reported that operation times ranged from 330 to $720 \mathrm{~min}$, EBL ranged from 100 to $500 \mathrm{ml}$, LOS ranged from 5 to 17.4 days, early minor complications from $17 \%$ to $62.5 \%$, major complications from $19 \%$ to $27 \%$ for neobladder series. The results of our study were comparable to the results of this review.

In the study on the timing of ePLND in open RC, the mean number of lymph nodes removed was lower in the group with ePLND performed before cystectomy than after cystectomy $(27.31 \pm 10.36$ vs. $30.87 \pm 8.3$, respectively, $p=0.041$ ). They evaluated the number of lymph nodes removed from eight regions. The number of lymph nodes removed was higher in the group with ePLND performed after RC for all regions. But only the number of lymph nodes removed from the presacral region was statistically significant. So they decided to re-check the presacral area for additional lymph nodes after cystectomy for patient who underwent ePLND before cystectomy.

The overall mean number of lymph nodes removed was $19.07 \pm 7.285$ in the present study. It was $23.75 \pm 3.454$ in group 1 and $13.71 \pm 6.873$ in group 2 ( $p=0.007)$. Canda et al. [18] (27 patients), Collins et al. [25] (43) Pruthi et al. [26] (100), Murphy et al. [27] (23), and Guru et al. [28] (67) reported mean LN yields of $24.8,21,19,16$ and 18 respectively in their RARC series. The overall number of lymph nodes removed was similar as in previous publications in experienced centers as the first robot-assisted intracorporeal neobladder series [18, 25-28]. The number of lymph nodes in group 2 was low compared to the literature, we think this is due to the cases in this group were our first intracorporeal RARC cases.

RARC is a difficult procedure with a steep learning curve and high morbidity. The number of centers which apply intracorporeal totally RARC and the number of patients are still limited in the literature.
An advantage of starting PLND before cystectomy is that the pedicle and tissue plan can be revealed more clearly; thus there was less bleeding and the procedure was performed more quickly. In addition, since the tissue was sent to the pathology laboratory as a whole, lymph node scanning started from the nodes in the proximal bladder to distally, and thus lymph node mapping could be performed.

In the present study, although the OT was comparable to the totally intracorporeal neobladder series in the PLND performed before the cystectomy group, it was longer than in the published series from experienced centers. We think that this is due to the fact that the number of our cases in totally intracorporeal RARC was only 15 . As the number of cases and our experience in totally intracorporeal RARC increase, we think that this period will be much shorter.

Since the bladder is not removed, dissection in the narrow pelvic area, especially in large bladder masses, can be considered as a disadvantage of performing PLND before cystectomy. In addition, presacral lymph node dissection cannot be performed clearly without bladder removal. To perform lymphadenectomy in the presacral region, initial mobilization of the sigmoid mesentery is essential and this process may become easier after cystectomy. Therefore, presacral lymph node dissection is performed separately after removal of the bladder and sent as a separate specimen as previously reported [15].

Limitations of this study include the fact that the number of cases was too low for statistical evaluation in our first totally intracorporeal RARC series. The second was that since our study was retrospective, PLND, cystectomy and urinary diversion times could not be compared separately; only total operation times were compared between the groups. The third limitation was that although the pathology was very suitable for regional evaluation of lymph nodes, the evaluation was done only as lymph node positivity, while the region of positive lymph nodes was not reported.

\section{Conclusions}

Our initial experience with performing ePLND before or after cystectomy in totally intracorporeal RARC appears to be favorable, with similar oncological re- 
sults and acceptable complication rates. However, although the number of cases is too low for statistical evaluation, it seems to be advantageous to perform ePLND before cystectomy in terms of LN number and operation time. Larger series of prospective randomized studies are needed to evaluate the effect of timing lymphadenectomy on operative outcomes.

\section{Conflict of interest}

\section{The authors declare no conflict of interest.}

\section{References}

1. Huang GJ, Stein JP. Open radical cystectomy with lymphadenectomy remains the treatment of choice for invasive bladder cancer. Curr Opin Urol 2007; 17: 369-75.

2. Moschini M, Zamboni S, Soria F, et al. Open versus robotic cystectomy: a propensity score matched analysis comparing survival outcomes. J Clin Med 2019; 8: 1192.

3. Nix J, Smith A, Kurpad R, et al. Prospective randomized controlled trial of robotic versus open radical cystectomy for bladder cancer: perioperative and pathologic results. Eur Urol 2010; 57: 196-201.

4. Desai MM, Gill IS, de Castro Abreu AL, et al. Robotic intracorporeal orthotopic neobladder during radical cystectomy in 132 patients. J Urol 2014; 192: 1734-40.

5. Dorin RP, Skinner EC. Extended lymphadenectomy in bladder cancer. Curr Opin Urol 2010; 20: 414-20.

6. Dhar NB, Klein EA, Reuther AM, et al. Outcome after radical cystectomy with limited or extended pelvic lymph node dissection. J Urol 2008; 179: 873-8.

7. Guijarro A, Hernández V, Llorente C. The anatomical limits and oncological benefit of lymphadenectomy in muscle invasive bladder cancer. Actas Urol Esp 2017; 41: 284-91.

8. Perera M, McGrath S, Sengupta S, et al. Pelvic lymph node dissection during radical cystectomy for muscle-invasive bladder cancer. Nat Rev Urol 2018; 15: 686-92.

9. Bi L, Huang H, Fan X, et al. Extended vs non-extended pelvic lymph node dissection and their influence on recurrence-free survival in patients undergoing radical cystectomy for bladder cancer: a systematic review and meta-analysis of comparative studies. BJU Int 2014; 113: E39-48.

10. Humphrey PA, Moch H, Cubilla AL, et al. The 2016 WHO classification of tumours of the urinary system and male genital organs - part B: prostate and bladder tumours. Eur Urol 2016; 70: 106-19.

11. Studer UE, Turner WH. The ileal orthotopic bladder. Urology 1995; 45: 185-9.

12. Wang GJ, Barocas DA, Raman JD, et al. Robotic vs open radical cystectomy: prospective comparison of perioperative outcomes and pathological measures of early oncological efficacy. BJU Int 2008; 101: 89-93.

13. Collins JW, Wiklund NP. Totally intracorporeal robot assisted radical cystectomy: optimizing total outcomes. BJU int 2014; 114: 326-33.
14. Holmer M, Bendahl PO, Davidsson T, et al. Extended lymph node dissection in patients with urothelial cell carcinoma of the bladder: can it make a difference? World J Urol 2009; 27: 521-6.

15. Ozen H, Ugurlu O, Baltaci S, et al. Extended pelvic lymph node dissection: before or after radical cystectomy? A multicenter study of the Turkish society of urooncology. KJU 2012; 53: 451-6.

16. Hayn MH, Hussain A, Mansour AM, et al. The learning curve of robot-assisted radical cystectomy: results from the International Robotic Cystectomy Consortium. Eur Urol 2010; 58: 197-202.

17. Thress TM, Cookson MS, Patel S. Robotic cystectomy with intracorporeal urinary diversion: review of current techniques and outcomes. Urol Clin North Am 2018; 45: 67-77.

18. Canda AE, Atmaca AF, Altinova $S$, et al. Robot-assisted nerve-sparing radical cystectomy with bilateral extended pelvic lymph node dissection (PLND) and intracorporeal urinary diversion for bladder cancer: initial experience in 27 cases. BJU Int 2012; 110: 434-44.

19. Schumacher MC, Jonsson MN, Hosseini A, et al. Surgery-related complications of robot-assisted radical cystectomy with intracorporeal urinary diversion. Urology 2011; 77: 871-6.

20. Johar RS, Hayn MH, Stegemann AP, et al. Complications after robot-assisted radical cystectomy: results from the International Robotic Cystectomy Consortium. Eur Urol 2013; 64: 52-7.

21. Khan MS, Elhage O, Challacombe B, et al. Analysis of early complications of robotic-assisted radical cystectomy using a standardized reporting system. Urology 2011; 77: 357-62.

22. Bochner BH, Dalbagni G, Sjoberg DD, et al. Comparing open radical cystectomy and robot-assisted laparoscopic radical cystectomy: a randomized clinical trial. Eur Urol 2015; 67: 1042-50.

23. Kim SP, Shah ND, Karnes RJ, et al. The implications of hospital acquired adverse events on mortality, length of stay and costs for patients undergoing radical cystectomy for bladder cancer. J Urol 2012; 187: 2011-7.

24. Lee R, Chughtai B, Herman M, et al. Cost-analysis comparison of robot-assisted laparoscopic radical cystectomy (RC) vs open RC. BJU int 2011; 108: 976-83.

25. Collins JW, Tyritzis S, Nyberg T et al. Robot-assisted radical cystectomy - description of an evolved approach to radical cystectomy. Eur Urol 2013; 64: 654-63.

26. Pruthi RS, Nielsen ME, Nix J, et al. Robotic radical cystectomy for bladder cancer: surgical and pathological outcomes in 100 consecutive cases. J Urol 2010; 183: 510-4.

27. Murphy DG, Challacombe BJ, Elhage O, et al. Robotic-assisted laparoscopic radical cystectomy with extracorporeal urinary diversion: initial experience. Eur Urol 2008; 54: 570-80.

28. Guru KA, Sternberg K, Wilding GE, et al. The lymph node yield during robot-assisted radical cystectomy. BJU Int 2008; 102: 231-4.

Received: 7.12.2019, accepted: 18.02.2020. 